\title{
Genome-wide CRISPR screens reveal synthetic lethal interaction between CREBBP and EP300 in diffuse large B-cell lymphoma
}

\author{
Man Nie ${ }^{1,2}$, Likun Du', Weicheng Ren ${ }^{1}$, Julia Joung ${ }^{3,4,5}$, Xiaofei Ye ${ }^{1}, X_{i}$ Shi $^{3}$, Sibel Ciftci ${ }^{1}$, Dongbing Liu ${ }^{6,7}$, Kui Wu ${ }^{6,7}$,
} Feng Zhang (D) $^{3,4,5}$ and Qiang Pan-Hammarström (i) ${ }^{1}$

\begin{abstract}
Diffuse large B-cell lymphoma (DLBCL) is the most common type of aggressive lymphoid malignancy and a highly heterogeneous disease. In this study, we performed whole-genome and transcriptome sequencing, and a genomewide CRISPR-Cas9-knockout screen to study an activated B-cell-like DLBCL cell line (RC-K8). We identified a distinct pattern of genetic essentialities in RC-K8, including a dependency on CREBBP and MDM2. The dependency on CREBBP is associated with a balanced translocation involving EP300, which results in a truncated form of the protein that lacks the critical histone acetyltransferase (HAT) domain. The synthetic lethal interaction between CREBBP and EP300 genes, two frequently mutated epigenetic modulators in B-cell lymphoma, was further validated in the previously published CRISPR-Cas9 screens and inhibitor assays. Our study suggests that integration of the unbiased functional screen results with genomic and transcriptomic data can identify both common and unique druggable vulnerabilities in DLBCL and histone acetyltransferases inhibition could be a therapeutic option for CREBBP or EP300 mutated cases.
\end{abstract}

\section{Introduction}

Diffuse large B-cell lymphoma (DLBCL) is one of the most common types of aggressive lymphoid malignancy. With the current standard immunochemotherapy, $\sim 30-40 \%$ of DLBCL patients still suffer from refractory disease or relapse ${ }^{1,2}$. Based on transcriptional profiles, two major subtypes of DLBCL have been defined: germinal centre B-cell like (GCB) and activated B-cell like $(A B C)^{1}$. Large-scale genome sequencing has further enabled the identification of several molecular subtypes of DLBCL based on genetic alterations affecting the proto-oncogenes

Correspondence: Feng Zhang (zhang@mit.edu) or

Qiang Pan-Hammarström (Qiang.Pan-Hammarstrom@ki.se)

'Department of Biosciences and Nutrition, Karolinska Institutet, Stockholm,

Sweden

${ }^{2}$ Department of Medical Oncology, Sun Yat-Sen University Cancer Center, State Key Laboratory of Oncology in South China, Collaborative Innovation Center for Cancer Medicine, Guangzhou, China

Full list of author information is available at the end of the article

These authors contributed equally: Man Nie, Likun Du

Edited by G. Melino
$B C L 2 /-6$ and $M Y C$, epigenetic modifiers and regulators in the B-cell receptor (BCR), nuclear factor- $\mathrm{kB}(\mathrm{NF}-\mathrm{kB})$, $\mathrm{NOTCH}$ and p53 signalling pathways ${ }^{3,4}$. The ABC subtype and selected molecular subtypes (C3 and $\mathrm{C} 5$ in Chapuy et al. $^{3}$; MCD and N1 in Schmitz et al. ${ }^{4}$; cluster with NOTCH1 mutations in Lacy et al. ${ }^{5}$ ) have been suggested to be associated with a poor prognosis. In addition, a new probabilistic classification tool, named LymphGen, was created to identify the genetic subgroups of DLBCL biopsy with therapeutic implications ${ }^{6}$. Recently, we have shown that hepatitis B virus (HBV)-related DLBCLs are associated with unique genetic and clinical features, as well as shorter overall patient survival, and may be considered a distinct subtype ${ }^{7}$. DLBCL is thus a highly heterogeneous disease and identification of genetic vulnerabilities that are specific to a subtype or subgroup of patients will aid in the development of novel targeted therapeutic strategies and improve clinical outcome.

One promising approach to systematically identify such genetic vulnerabilities is through genome-wide CRISPR-

\section{(c) The Author(s) 2021}

(c) (i) Open Access This article is licensed under a Creative Commons Attribution 4.0 International License, which permits use, sharing, adaptation, distribution and reproduction cc) in any medium or format, as long as you give appropriate credit to the original author(s) and the source, provide a link to the Creative Commons license, and indicate if changes were made. The images or other third party material in this article are included in the article's Creative Commons license, unless indicated otherwise in a credit line to the material. If material is not included in the article's Creative Commons license and your intended use is not permitted by statutory regulation or exceeds the permitted use, you will need to obtain permission directly from the copyright holder. To view a copy of this license, visit http://creativecommons.org/licenses/by/4.0/. 
Cas9 screening ${ }^{8}$, which has been used in a range of contexts to discover genetic dependencies and vulnerabilities in cancer cells ${ }^{9-11}$. In the context of B-cell malignancy, CRISPR-Cas9 screens have already provided valuable results for understanding the mechanisms of tumorigenesis $^{12,13}$ as well as discovery of drugs that might enhance tumour antigen presentation to $\mathrm{T}$ cells ${ }^{14}$.

Dysregulation of epigenetic modulators is frequently observed in lymphomas. EZH2, KMT2D, MEF2B, genes encoding linker histone $\mathrm{H} 1$ proteins, as well as CREBBP, the histone acetyltransferase (HAT) encoding gene and its paralogue $E P 300$, are among the most frequently mutated genes in B-cell lymphomas ${ }^{15-17}$. Both CREBBP and EP300 are large multidomain proteins that, in addition to their catalytic HAT domain, contain bromodomains (BRDs) that bind acetylated histones and are required for chromatin binding ${ }^{18}$. Despite the sequence homology and functional similarities between CREBBP and $E P 300^{19}$, monoallelic germline lesions in either gene may cause a severe phenotype, i.e., Rubinstein-Taybi syndrome ${ }^{20}$. In B-cell lymphomas, heterozygous somatic mutations in either gene may result in haploinsufficiency and these mutations have been described as mutually exclusive in most cases ${ }^{17}$. Genetic alterations in CREBBP are, however, more frequently observed in these disorders than deficiency of EP300. Functional screening using a small interfering RNA library suggested that EP300 is a specific synthetic lethal gene in CREBBP-deficient lung cancer cells $^{21}$. A recent study further showed that the synthetic lethal interaction between CREBBP and EP300 was obtained in both normal germinal centre $B$ cells and CREBBP-mutant DLBCL cells, raising the possibility of targeting EP300 in the treatment of CREBBP-mutated tumours ${ }^{22}$.

In this study, we performed whole-genome and transcriptome sequencing to characterize an $\mathrm{ABC}$-like DLBCL cell line (RC-K8) established from peritoneal effusions of a patient with terminal, refractory-stage disease $^{23}$. We also used an unbiased, genome-wide CRISPR-Cas9 loss-of-function screening approach to study genetic dependencies in this cell line. For comparison, we have furthermore evaluated the published CRISPR-Cas9 screens from a pan-cancer study and sets of DLBCL cell lines. We identified a distinct pattern of genetic essentialities in $\mathrm{RC}-\mathrm{K} 8$, including a specific dependency on CREBBP in the context of EP300 deficiency. We also observed the essentiality of EP300 in several DLBCL cell lines harbouring mutation or copy number loss in CREBBP. Our results confirmed and extended the previous finding on synthetic lethal interaction between the two important epigenetic modulators, and suggest that the dependency of the remaining HAT function is a druggable vulnerability in CREBBP- or EP300-deficient DLBCLs.

\section{Results}

Characterization of the genome and transcriptome of RCK8 cells

RC-K8 cell line was established from a patient with lymphoma, described at the time as histiocytic lymphoma (terminal and refractory stage) ${ }^{24}$, and later was assigned to the GCB subtype of DLBCL ${ }^{25}$. However, more recent studies on this cell line suggested a constitutive NF- $\kappa B$ signalling, a feature that is usually associated with the $\mathrm{ABC}$ subtype ${ }^{26}$. Cytogenetic analysis and fluorescence in situ hybridization analysis have identified the karyotype of this cell line and a translocation involving the BCL6 gene $^{27,28}$. To further characterize the RC-K8 cell line, we performed whole-genome and transcriptome sequencing (RNA sequencing (RNA-seq)) on DNA and RNA samples derived from this cell line. Based on the distribution of genomic sequence coverage, we discovered trisomy of chromosome 7 and gain of part of chromosomes 5, 13 and 20 (Fig. 1A and Supplementary Fig. S1), and estimated copy number gains of $M Y C$ and $N O T C H 1$, as well as amplification of REL (Supplementary Table S1). In addition, RC-K8 cells were estimated to have copy number losses of $C D 70$ and $U B E 2 A$, and two cohesion-related genes, STAG2 and SMC1A (Supplementary Table S1). We further identified 384 genes with nonsynonymous mutations $(n=436)$, including genes involved in DNA damage response and repair (RAD21, TP63, TP73, and XRCC6), $\mathrm{BCR} / \mathrm{NF}-\mathrm{KB}$ signalling (TNFAIP3 and NFKBIA), and transcription factors important for B-cell development (IKZF1 and IKZF3) (Supplementary Table S2). Sequencing data showed that the RC-K8 cell line harbours a largely normal TP53 gene with a benign P72R polymorphism but with a relatively high expression level of its negative regulator MDM2 (fragments per kilobase of transcript per million mapped reads: 46.55), as estimated by RNA-seq. Through whole-genome sequencing (WGS), we also identified and mapped structural variants to base pair resolution including translocations involving $I G H$ and BCL6 (with different translocation partners), as well as a balanced translocation between chromosomes 22 and 6 in RC-K8 (Fig. 1B), which resulted in a C-terminal truncation of EP300 that has been previously reported as $E P 300 \Delta C 1047^{23,29}$. RNA-seq analysis further demonstrated dominant allelic expression of the truncated form of EP300 (Fig. 1C), which is encoded by exons $1-17$ of $E P 300$ and fused to 25 amino acids encoded by intronic sequences of the $B C K D H B$ gene on chromosome 6 , resulting in the loss of two critical domains of this protein, i.e., the BRD and HAT domains ${ }^{23}$. Finally, based on the gene expression data, RC-K8 indeed showed an ABC, rather than GCB gene expression signature (Supplementary Fig. S2) ${ }^{12}$. In summary, by sequencing the genome and transcriptome of $\mathrm{RC}-\mathrm{K} 8$, we have characterized this cell line as an $\mathrm{ABC}$-like DLBCL line, with unique genetic 


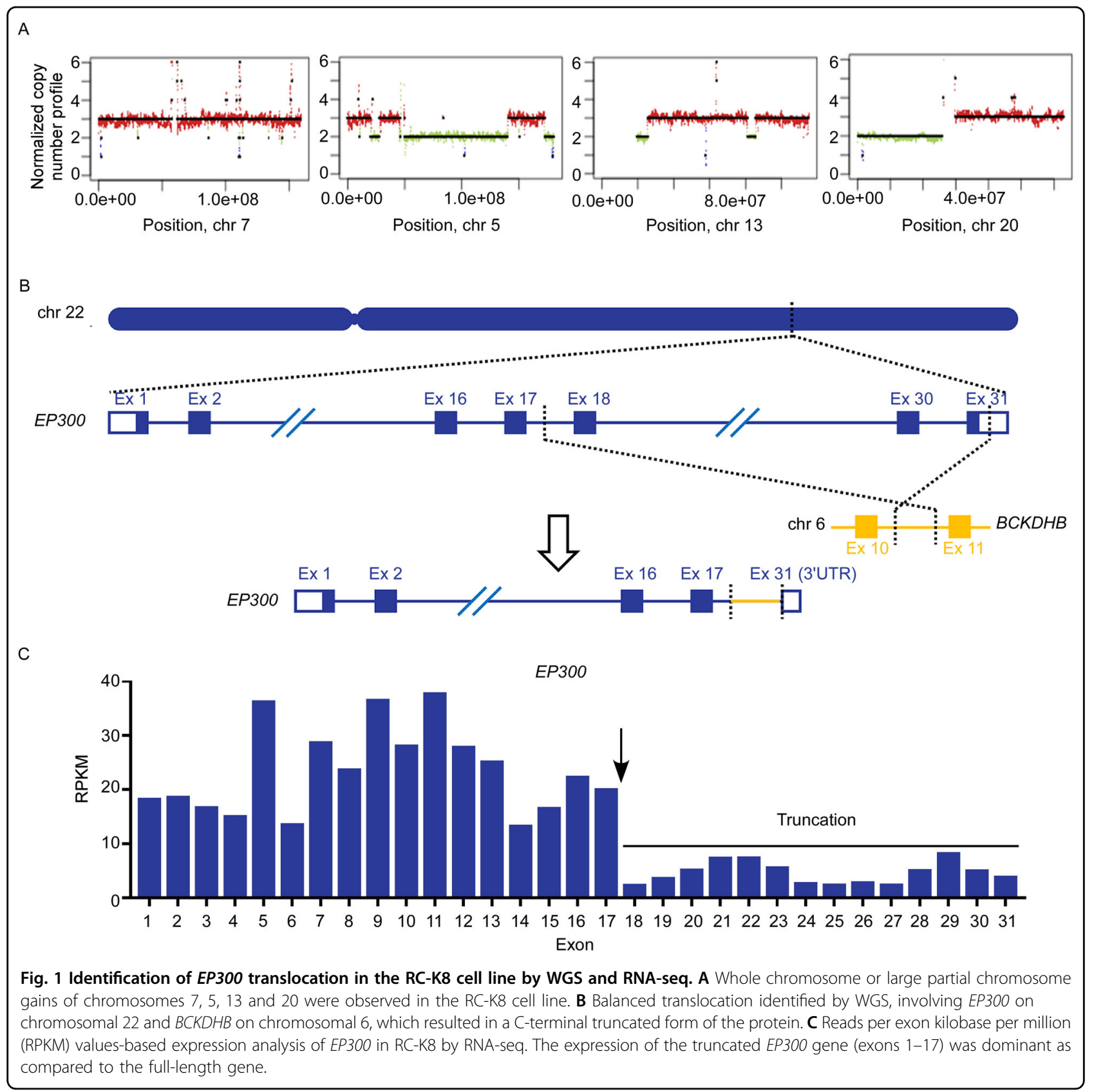

alterations, including a translocation that resulted in a truncation of EP300.

\section{Genome-scale CRISPR-Cas9 knockout screening reveals essential genes and pathways in RC-K8 cells}

To investigate the cancer dependencies related to the genetic background of the RC-K8 cell line, we performed a functional genetic screen by introducing the genomewide pooled CRISPR-Cas9 knockout library (detailed in "Methods"). A Cas9-expressing RC-K8 cell line (RC-K8Cas9) was first generated and subsequently transduced by the pooled lentiCRISPRv2 library, which targets 19,050 human genes with more than 100,000 unique single-guide RNAs (sgRNAs). By deep sequencing the amplified sgRNA library, we identified depleted guide RNAs (gRNAs) caused by dropout of cells bearing related genetic perturbations, which reflects the importance of the targeted genes for cell growth or proliferation.

We then applied the optimized MAGeCK-based scoring system (detailed in "Methods") to estimate the relative effects of gene knockouts during the 28-day time course of the CRISPR screen on RC-K8 cells (Fig. 2A). Based on both the read-count distribution and the standardized CRISPR scores, we observed no significant depletion at 


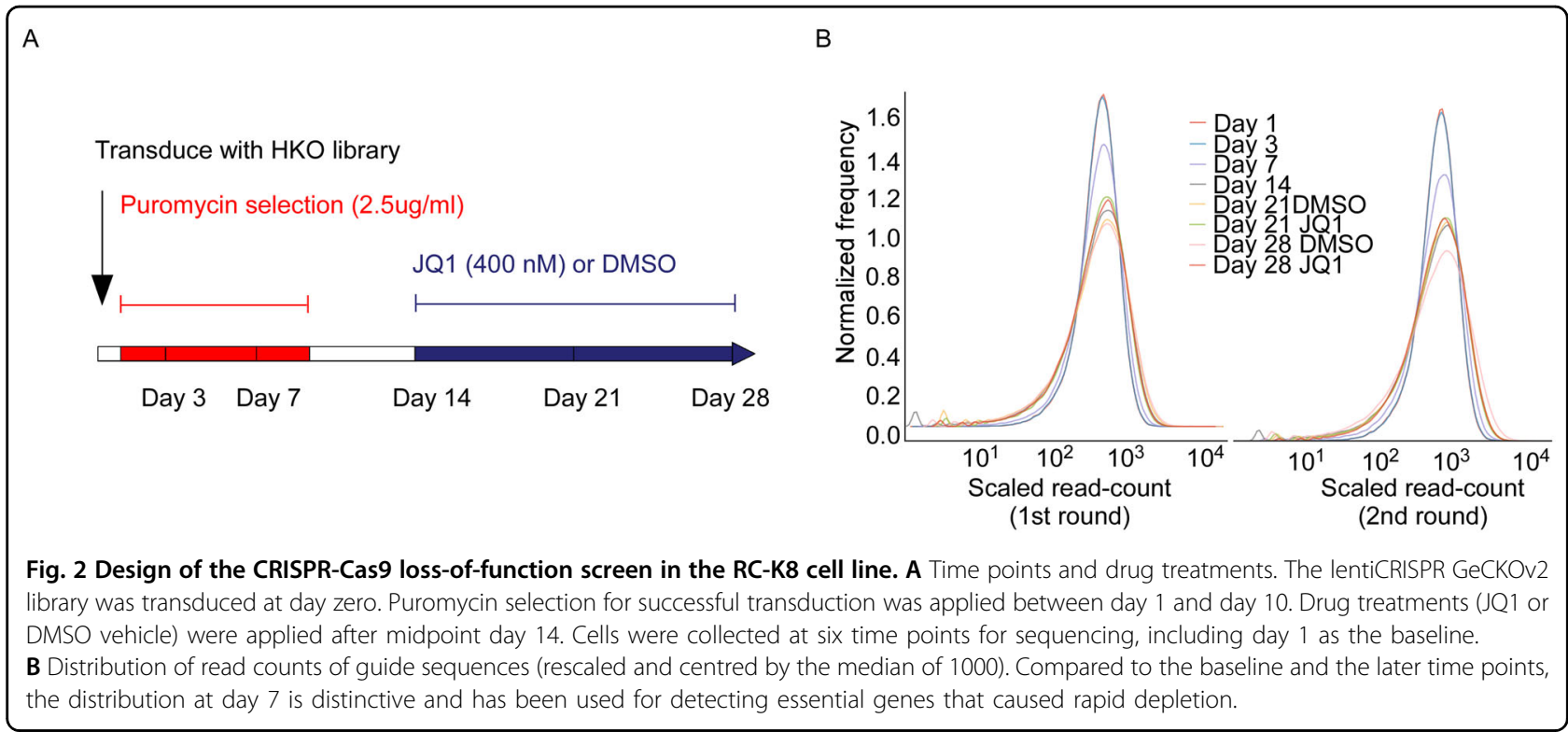

day 3 after transduction (Fig. 2B). In contrast, at day 7, we started to observe a rapid depletion of cells bearing knockouts of core fitness genes related to ribosome, spliceosome, proteasome and cell cycle regulation (Fig. 3) $9,10,30,31$. At day 7 , the top candidates for core fitness genes also included cell-type specific essentialities (Supplementary Table S1). Several DLBCL essential genes, as estimated from the previous two screens ${ }^{12,13}$, were detected early at day 7, including FOXO1, IRF4 and $S F 3 B 1$, whereas some appeared later than day 14, e.g., TAF1, MLL2, RHOA and YY1 (Supplementary Table S1). Specifically, for RC-K8 cells, which carry a largely normal TP53 gene locus, MDM2 was one of the most significant essential genes detected at day 7, demonstrating its critical role in suppressing TP53-induced cell death (Fig. 3 and Supplementary Table S1). When re-analysing the CERES data set (CRISPR screens on 341 cancer cell lines) ${ }^{32}$, we indeed observed that cancer cell lines with wild-type TP53 in general are more dependent on MDM2 and MDM4 (Supplementary Fig. S3). CCND3, which is associated with both TP53 signalling and cell cycle regulation pathways, exhibited essentiality and specificity to the cell line (Fig. 3). Similarly, CREBBP was also identified at day 7 as one of the top hits, showing its essentiality in the context of EP300 loss-of-function (Fig. 3).

On the axis of positive selection, the effects of knocking out growth-suppressing genes were generally accumulative. We observed a significant anti-proliferative effect of the classical tumour suppressor TP53 and several associated regulators/effectors, such as SESN2 and BAI1 (Fig. 3). Knockouts of some "cancer drivers" of DLBCL, including BCL2, BCL6, NFKBIA and CD70, showed only modest promotional effects on cell proliferation (Supplementary Table S1).
We validated several top-ranking genes (essential genes $M D M 2$ and $C R E B B P$, tumour suppressor gene TP53) from genome-wide screening by single-knockout experiments (Supplementary Fig. S4A-D) and further confirmed the rapid and complete responsiveness to MDM2 inhibition by nutlin-3 (Supplementary Fig. S4E).

\section{Late time-point depletion identifies different essential pathways in RC-K8}

The standardized CRISPR scores at day 7 exhibited a weak correlation (Pearson's $r<0.25$ ) with the average CERES scores (representing general essentialities in 341 cancer cell lines). In contrast, the cumulative effects at later time points correlated more strongly with the CERES scores, with the highest correlation at day 28 (Pearson's $r>0.48$ ) (Supplementary Fig. S5 and Table S1). We surmise that rapid depletions were caused by genetic lethality, whereas delayed dropouts were associated with impaired cellular function or fitness. We hypothesize that essential genes exhibiting different rates of depletion may participate in complementary pathways. To test this hypothesis, based on the ranks of CRISPR scores, we performed gene set enrichment analysis (GSEA) on 50 hallmark pathways (Fig. 4 and Supplementary Table S3). We observed that the most differentially enriched pathways between early and late time points (day 7 vs. day 28) were the apoptosis and oxidative phosphorylation (OXPHOS) pathways (Fig. 4). Compared to normalized enrichment scores (NES) of the 341 cell lines from the CERES data set, the rapid depletion of apoptosis-related genes showed cell-type specificity (Fig. 4), where BCL2L1, $C R E B B P$ and WEE1 were among the most significant genes (Supplementary Table S1). On the other hand, depletions associated with OXPHOS genes showed a 


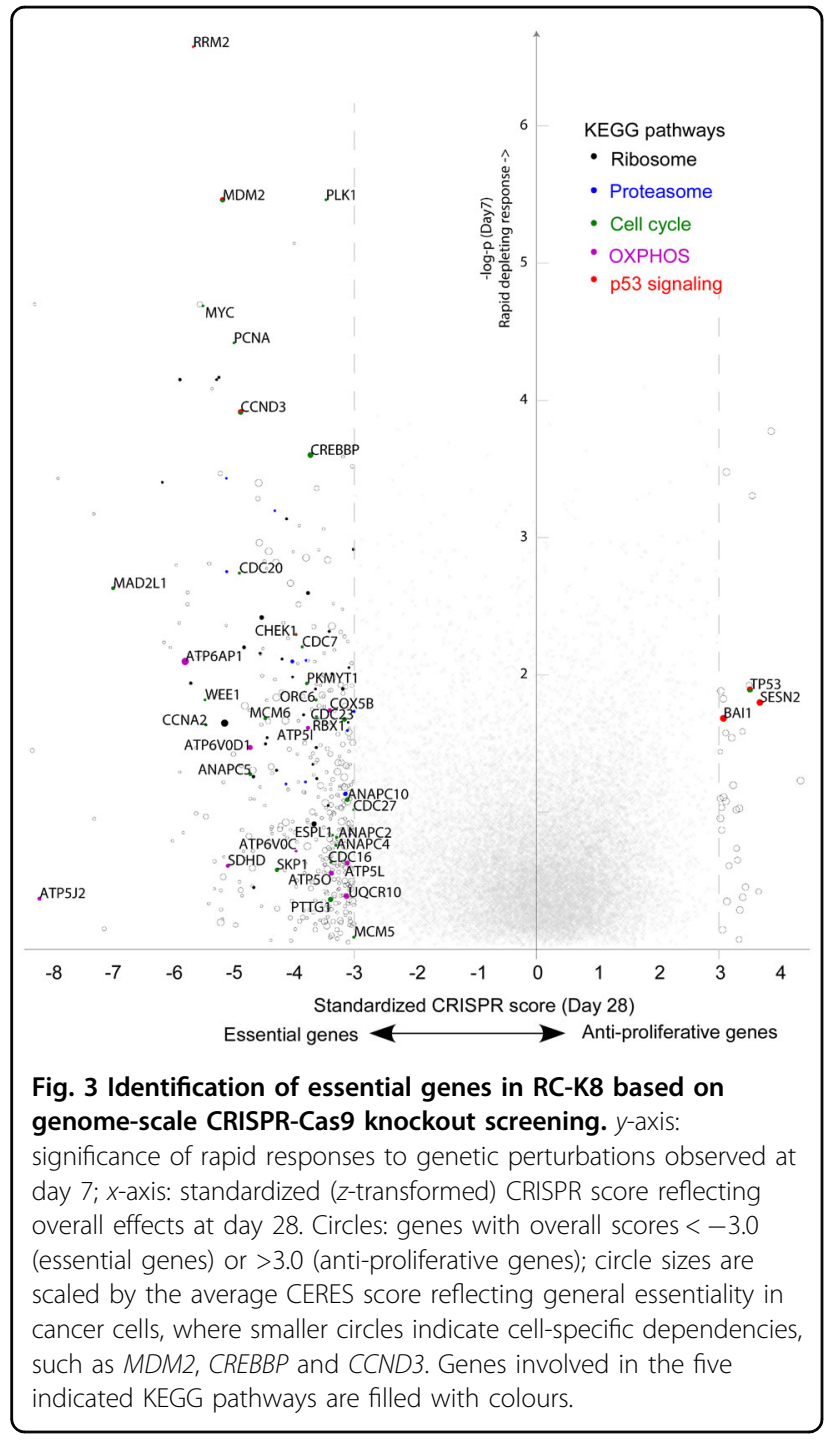

gradual increase over time (Fig. 4), suggesting that growth suppression caused by interrupted energy metabolism is slow and cumulative.

The data also allowed us to investigate, within the same analysis, the relative effects associated with JQ1 (a BET inhibitor) treatment during the screening process (Supplementary Table S4). BET inhibition by JQ1 has been suggested to trigger cell cycle arrest followed by apoptosis or senescence ${ }^{33}$, and we indeed observed a long-term growth-suppressive effect in the JQ1-treated cells. We found no significant enrichment of positively selected genes (those possibly causing resistance to the JQ1 treatment). Nonetheless, several negatively selected genes were found to be associated with the mitogen-activated protein kinase signalling pathway, including $P A K 2$, LAMTOR3 and MAPKAPK2 (Supplementary Table S1). Notably, the top candidate gene, $P A K 2$, was non-essential in RC-K8 cells in general but was selectively depleted in

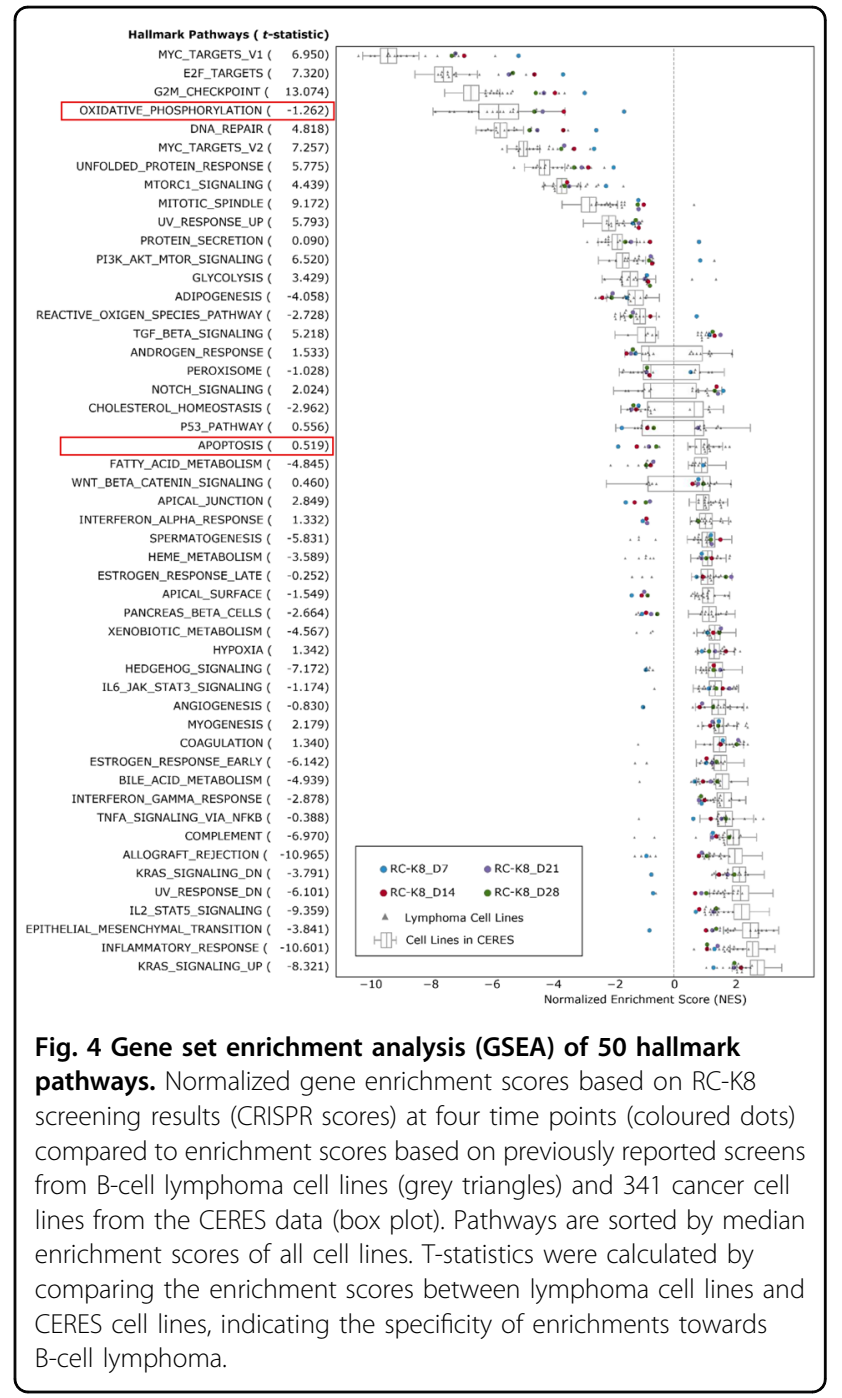

the JQ1-treated cells. This may indicate that the loss of PAK2 could sensitize RC-K8 cells to JQ1 treatment

\section{DLBCL cells with either EP300 or CREBBP mutations are sensitive to HAT domain}

By re-analysing a previously published DLBCL cohort (275 patients) ${ }^{7}$, we observed that mutations were more frequently identified in CREBBP than in EP300 (11.7\% vs. $3.3 \%$, Fig. $5 \mathrm{~A}$ ), which is in agreement with previous studies $^{17}$. Notably, over $70 \%$ of these mutations affected the HAT domain (Fig. 5A and Supplementary Table S5). In most of the affected cases, either CREBBP or EP300 was mutated, but we did identify several cases with potentially oncogenic mutations, affecting both the genes (Supplementary Table S5).

Based on re-evaluating and ranking the CRISPR scores of the reference datasets (the CERES data set on 341 cancer cell lines and two previous published screens on DLBCL cell lines) $)^{12,13,32}, C R E B B P$ and EP300 seemed to 


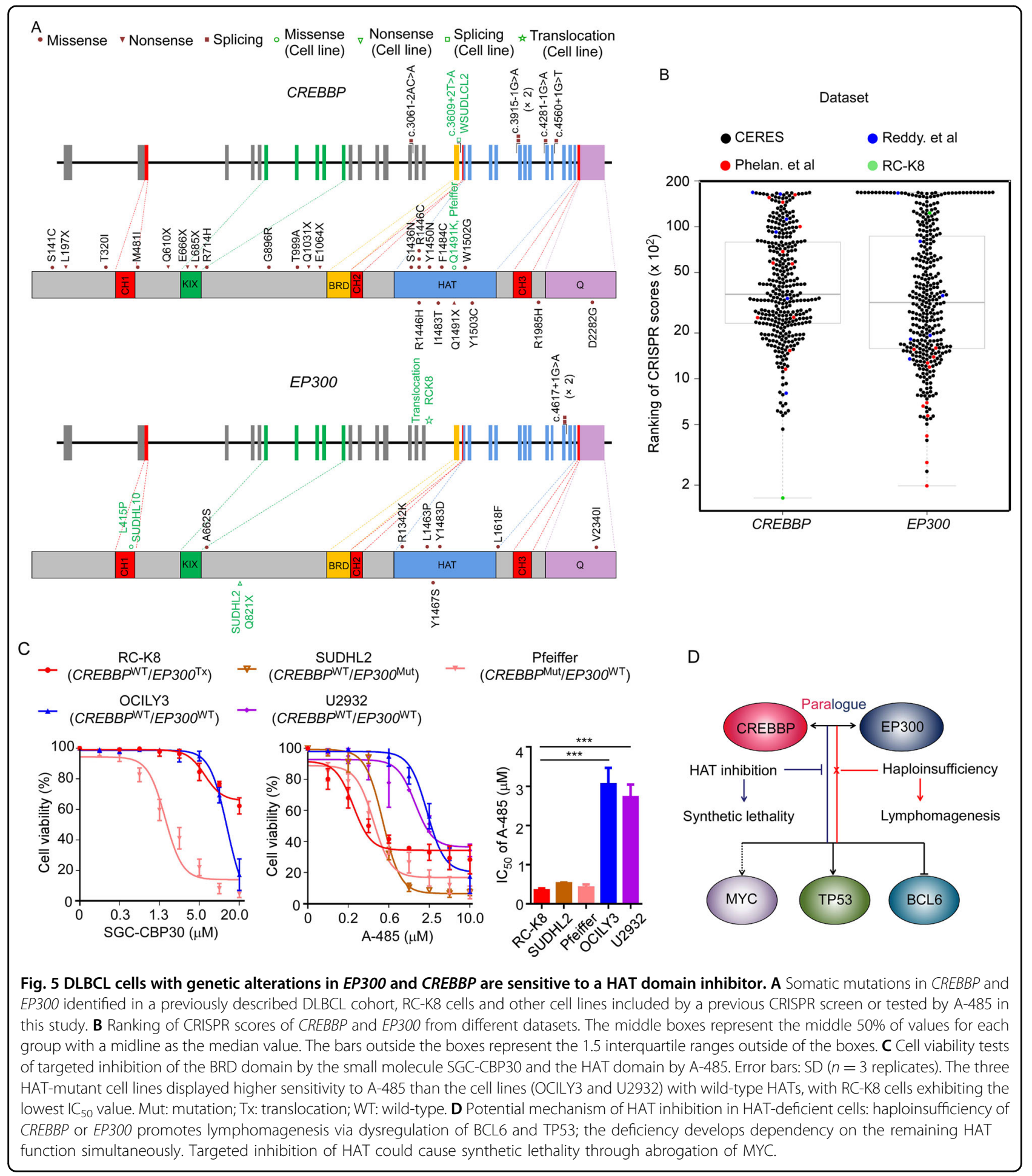

be essential in a small set of cell lines (Fig. 5B). Among the DLBCL cell lines analysed, CREBBP is almost uniquely essential in RC-K8 cells, whereas EP300 is more frequently identified as an essential gene (Fig. 5B and Supplementary Table S6), which may reflect the different mutational rates in these genes in DLBCL patients. Importantly, two GCB- like DLBCL cell lines that were highly sensitive to EP300 knockout harboured mutations (WSUDLCL2) or copy number variation (CNV) loss (SUDHL5) in CREBBP (Fig. 5B and Supplementary Table S6).

It has previously been reported that BRDs are also critical in sustaining the proliferation of lymphoma cells via 
epigenetic regulation. Therefore, we tested the CREBBP/ EP300-specific BRD inhibitor SGC-CBP30 in RC-K8 cells and observed a limited effect on proliferation (Fig. 5C), which prompted us to further perform cell viability tests using a newly reported catalytic inhibitor (A-485) that targets the HAT domain of both CREBBP and $E P 300^{34}$. RC-K8 cells responded to A-485 treatment, with the lowest IC50 value $(0.42 \mu \mathrm{M})$ compared to four other DLBCL cell lines tested (Fig. 5C and Supplementary Table S6). In general, the three cell lines with CREBBP and/or EP300 mutations showed a higher sensitivity than the two wild-type cell lines for these genes (OCILY3 and U2932) (Fig. 5C), suggesting that dependency of the remaining HAT function is a druggable vulnerability in DLBCL cells with either CREBBP or EP300 genetic alterations (Fig. 5D).

\section{Discussion}

In this study, we performed a genome-wide CRISPRCas9 loss-of-function screening and identified CREBBP as a vulnerability specific to the ABC-like DLBCL cell line $\mathrm{RC}-\mathrm{K} 8$, which harboured a translocation that disrupts the EP300 gene. CREBBP is one of the most frequently mutated genes in non-Hodgkin lymphoma (NHL) and usually plays a tumour-suppressing role through epigenetic regulation ${ }^{35}$. Such dependency on CREBBP can be best explained by a synthetic lethality associated with the loss-of-function of its paralogue EP300 (Fig. 5D) ${ }^{21}$. As reported in multiple myeloma ${ }^{36}$, the BRD of $C R E B B P$ is essential for regulating the DLBCL essential gene IRF4. Although IRF4 also exhibited essentiality in our CRISPR screen with RC-K8 cells, the BRD inhibitor CBP30 did not show efficacy. In contrast, A-485, a potent catalytic inhibitor of HAT domains ${ }^{34}$, showed high efficacy in this EP300-deficient cell line. The results suggested that the HAT domain of CREBBP is linked to essentiality in RCK8 cells. By re-evaluating the previously published CRISPR-Cas9 screens from a pan-cancer study and sets of DLBCL cell lines, we also identified a genetic essentiality of EP300 or CREBBP gene in a small subset of cancer cell lines, including a few DLBCL cell lines that are dependent on EP300 and carry CREBBP mutation, copy number loss or translocation. We further observed differences in the $\mathrm{IC}_{50}$ values of $\mathrm{A}-485$ in five lymphoma cell lines, indicating a potential correlation between CREBBP/EP300 deficiency and sensitivity to HAT inhibitors. Our results thus suggest that targeting the remaining HAT function may hold therapeutic potential for B-cell lymphomas with deficiency in either CREBBP or EP300.

The genetic features of the RC-K8 cell line showed some similarity to the $\mathrm{BN} 2$ (based on BCL6 fusions and NOTCH1 CNV changes) ${ }^{4}$ or the $\mathrm{C} 1$ (based on BCL6 structural variants and mutations of $\mathrm{NOTCH}$ signalling pathway components) molecular subtype ${ }^{3}$ but also have distinct genetic features. We also noted that it resembled what we have discovered previously in DLBCL samples associated with $\mathrm{HBV}$ infection ${ }^{7}$, with translocations in $B C L 6$, copy number changes in NOTCH1 and $C D 70$, and mutations in ZFP36L1, SGK1, IKZF3, TP63 and TP73. It has been shown that the HBV protein $\mathrm{HBx}$ can directly interact with $C R E B B P / E P 300$ and facilitate the recruitment of the complex onto CREB-responsive promoters, upregulating downstream oncogenes ${ }^{37}$. This was supported by the observation that the CREBBP-targeted genes were significantly upregulated in $\mathrm{HBsAg}^{+}$tumours compared to $\mathrm{HBsAg}^{-}$tumours (Supplementary Fig. S6). Targeting the HAT functions of $C R E B B P / E P 300$ can thus be a new direction in developing effective treatment for $\mathrm{HBV}$-associated DLBCL patients, who usually have a poor response to the current therapy ${ }^{7}$.

In addition to $C R E B B P$, we also identified the cell linespecific essentiality of $M D M 2$ in $\mathrm{RC}-\mathrm{K} 8$, which has an increased level of expression of $M D M 2$ and a largely unaffected TP53 gene. Accordingly, the screen also showed a strong positive selection of TP53 in RC-K8 cells. TP53 is mutated in $~ 50 \%$ of human cancers and is one of most studied tumour suppressors ${ }^{38}$. In other tumours, including the majority of DLBCLs, however, TP53 is in its wild-type form and targeting the negative regulators of TP53, such as MDM2, may be a promising approach ${ }^{39}$. A recent CRISPR-Cas9 screen also identified druggable dependencies in TP53 wild-type Ewing sarcoma, including $M D M 2, M D M 4, U S P 7$ and $P P M 1 D^{40}$.

RC-K8 cells have a normal functional TP53, which is capable of triggering DNA damage responses to CRISPRCas9 editing during the screening process ${ }^{41}$. Consequently, the calling of essential genes through a depleting effect might be affected by TP53-induced apoptosis or cell cycle arrest. We therefore used a time-course experimental design to distinguish between essentiality and DNA damage response, as cells carrying dispensable knockouts may recover from the perturbation and then grow back. We observed that many such genes exhibited a rapid depleting effect but were not ultimately essential (Supplementary Table S1). During the time course of the CRISPR screen, we also observed a delayed depleting effect of many essential genes and pathways, notably the OXPHOS-related genes. Such delayed essentialities of metabolic pathways are repeatedly observed in CRISPRbased genetic screens ${ }^{30,42}$. One possible reason for the delayed depletion is the poor correlation between mRNA and protein expression, which has been observed in proteomics studies ${ }^{43}$. Thus, highly expressed genes may provide a buffer to CRISPR-induced perturbations and cause false negative results in essentiality calling. The time-course information, analysed by our optimized method, may help to detect such delayed essentialities in cancer cells. 
Our unbiased, genome-wide, time course-based CRISPR-Cas9 screen revealed a number of cell linespecific vulnerabilities, such as CREBBP and MDM2, and a delayed metabolic dependency of OXPHOS genes. By re-analysing the previously published CRISPR-Cas9 screens, we also identified a genetic essentiality of EP300 in additional DLBCL cell lines. Considering the high mutation rates of CREBBP and EP300 in DLBCL and follicular lymphoma (FL), the two most common types of NHL, and the prognostic value of these two genes in FL (M7-FLIPI $)^{44}$, the findings of our study provide insights for the development of more effective targeted therapies as well as novel combination treatments that may benefit a large group of patients. It is also important to point out that DLBCL is a highly heterozygous disease, and that each tumour carries a unique combination of genetic alterations, affecting multiple functional pathways. Characterization of the "core fitness" gene for cancer cells and DLBCL essential genes might be informative for prioritization of therapeutic targets ${ }^{11}$; however, integration of genetic/transcriptomic data with unbiased functional screening will still be needed to identify the most effective targeted therapy for individual/subgroup of patients.

\section{Methods}

\section{Cell culture}

DLBCL cell lines RC-K8, OCILY3, SUDHL4 and Pfeiffer were purchased from the Leibniz-Institute DSMZ (Braunschweig, Germany) or the American Type Culture Collection (Manassas, USA). A cell line authentication test was performed for the RC-K8 cell line using PCR singlelocus technology (Eurofins Genomics, Germany). U2932 was kindly provided by Dr. G. Enblad's research group (Uppsala University). All cell lines were cultured in RPMI 1640 medium (Invitrogen, Carlsbad, USA) supplemented with 10\% fetal bovine serum (Gibco, Invitrogen, Paisley, UK).

\section{Whole-genome sequencing}

DNA from RC-K8 cells was sequenced using the BGI500 sequencing platform (BGI-Shenzhen, Shenzhen, China). After quality control, $100 \mathrm{bp}$ paired-end clean reads corresponding to $30 \times$ sequencing coverage were acquired. Sequence alignment to the reference genome (hg19) and mutation calling were performed by applying the "best-practice" GATK workflow. Mutations were excluded based on the following criteria: (1) a frequency higher than 0.01 in the ExAC (all and Asian), 1000 Genomes (all and Asian) and ESP6500 databases; (2) minor allele frequency $<10 \%$ or $>90 \%$; and (3) a reference single nucleotide polymorphism ID (rs) number in dbSNP build 147. Structure variations in the RC-K8 cell line were detected using Manta ${ }^{45}$ as described previously ${ }^{46}$ and copy number variations were estimated using ControlFREEC $^{47}$.

\section{Transcriptome sequencing}

Total RNA of RC-K8 was sequenced at BGI-Shenzhen using the Illumina HiSeq 2000 platform. Raw sequencing reads with adaptors, with $>10 \%$ unknown bases or with $>50 \%$ low-quality bases in one read, were filtered out. Clean reads were aligned to the reference transcriptome (Hg19) by SOAP2 and SOAPfusion for detecting genefusion events. Gene expression levels were reported as reads per exon kilobase per million.

\section{CRISPR-Cas9 screen with pooled lentiCRISPRv2 library}

Following a previously described procedure ${ }^{48}$, a cell line with stably expressed Cas9 (RC-K8-Cas9) was generated and was subsequently transduced by the pooled lentiCRISPRv2 library (multiplicity of infection $\sim 0.3$ ). One hundred fifty million cells were collected $24 \mathrm{~h}$ after transduction as the baseline and the remaining cells were cultured under $2.5 \mu \mathrm{g} / \mathrm{ml}$ puromycin (Sigma, Darmstadt, Germany) selection for 10 days. At days 3, 7 and 14, 110, 60 and 60 million cells were collected, respectively, whereas 120 million cells were kept in culture after day 14 and treated with either JQ1 (400 nM) (Tocris Bioscience, Abington, UK) or dimethyl sulfoxide (DMSO) for two additional weeks. For each treatment, 60 million cells were collected at two time points: day 21 and day 28 . Genomic DNA was extracted from cells collected at different time points using the Blood \& Tissue Kit (Qiagen, Hilden, Germany). Guide sequences were PCR-amplified and sequenced at the Broad Institute using the Illumina NextSeq sequencer following a reference protocol ${ }^{48}$. The screening procedure was performed independently in two replicate experiments.

\section{Preprocessing of CRISPR-Cas9 screen data}

A modified preprocessing step derived from the original protocol (count_spacers.py script) ${ }^{48}$ was applied, trimming raw reads in FastQ format by the spacer sequences from both ends (3': GTTTT and 5': CGAAACACC) using CutAdapt (v1.8.3 Martin, 2011). One mismatch was allowed in the $5^{\prime}$ sequence (parameter -e 0.12). The trimmed reads with a length of 18-21 bp were mapped to the FASTA library of guide sequences (GeCKO v1/v2, Addgene.org) using Bowtie $2^{49}$. To increase the specificity of read-count data, we mapped the library against the human genome sequence and excluded guide sequences targeting non-protein coding regions (named with "mir-" or "let-" tags) or having alternative alignments (filtered by "XS" and "NM" tags in the bowtie2 result). After filtering, read-count data were normalized by the median count of each individual experiment.

\section{Gene essentiality estimation}

The MAGeCK maximum-likelihood estimation (MLE) algorithm was used to estimate the relative screening 
effects ( $\beta$-scores or modified log fold changes) in both negative and positive directions, i.e., depletion and enrichment. In the design matrix of the MAGeCK analysis, samples were binary coded in different groups based on time points, batches and drug treatments (Supplementary Table S4). Samples from day 1 were labelled the baseline (zero) in all grouping conditions. As a result, one $\beta$-score was calculated by MLE for each gene in each condition. To resolve the CRISPR screening effect with the time course, $\beta$-scores were fit by linear interpolation curves along the time axis ( $k$ days). For every gene, given that day 1 is the baseline: $\beta(1)=0$, the integral of $\beta$-scores at a time point $(k)$ was used as the proxy of the overall CRISPR screening effect: $S_{k}=\int_{1}^{k} \beta(t) d t$. To normalize the integral scores, null distributions of scores were estimated based on a null set of read-count data generated by permutating the identities of sgRNA sequences. Null scores were calculated by MAGeCK-MLE following the same procedure and then fitted by Gaussian distributions at each time point. Subsequently, the original integral scores were standardized by the mean and SD of the null distribution: $Z_{\text {gene-k }}=\frac{S_{\text {gene. }}-\bar{S}_{\text {null.k }}}{\sigma\left(S_{\text {null.k }}\right)}$.

To test the validity of the scoring method, two reference datasets (HT-29 ${ }^{30}$ and CERES ${ }^{32}$ ) were used for benchmarking, i.e., the HT-29 data set (colon cancer cell line, with multiple time points, day 3 to day 25) and the CERES data set (341 cancer cell lines, with 1 time point). The Pearson's correlation coefficients between the estimated CRISPR effects and the general essentialities of the average CERES scores served as the figure-of-merit for overall performance at each time point. In addition, two previously published CRISPR screens were used to characterize essential DLBCL genes ${ }^{12,13}$. To compare the results from different datasets, for each cell line, we also ranked the CRISPR scores for 16,821 genes that were studied in all samples.

GSEA was performed by using the pre-ranked GSEA approach $^{50}$. The 50-hallmark gene sets were used in GSEA, while weights were set to zero (classic method) ${ }^{51}$. One thousand permutations were used and NES were used for comparisons between datasets.

\section{Single-gene knockout experiments}

Two to three sgRNAs for each of the targeted genes and nontargeted controls were chosen from the lentiCRISPRv2 library and individually cloned into the plasmid backbone of the sgRNA library. Lentiviruses were subsequently produced in $293 \mathrm{~T}$ cells for each selected sgRNA. In each single-gene knockout experiment, three million RC-K8-Cas9 cells were transduced and then selected by puromycin $(2.5 \mu \mathrm{g} / \mathrm{ml})$. The growth of cells was monitored by counting the numbers of living cells at five time points (days 1, 3, 5, 9 and 14). DNA was extracted from cells collected at day 5 to verify the desired targeting (introduction of loss-of-function insertions or deletions) of each selected sgRNA by SURVEYOR assay (Integrated DNA Technologies, Coralville, USA) and sequencing ${ }^{48}$.

\section{Cell viability assay}

A total of $2 \times 10^{4}$ cells were seeded in a volume of $100 \mu \mathrm{L}$ per well with vehicle or the indicated concentrations of drugs (nutlin-3, Santa Cruz Biotechnology, USA; SGC-30, Sigma-Aldrich; A-485, Selleckchem). All drugs were dissolved in DMSO. At $72 \mathrm{~h}$ post treatment, $20 \mu \mathrm{l}$ per well of CellTiter 96 AQueous One Solution Reagent (Promega, Madison, USA) was added. After incubation of the plates for $4 \mathrm{~h}$ at $37^{\circ} \mathrm{C}$, cell viability was measured by comparing the absorbance (A) at $450 \mathrm{~nm}: A_{\text {treatment }} /$ $A_{\text {control }} \times 100 \%$. Each experiment was independently repeated at least three times.

\section{Statistics}

$P$-values were calculated by Student's $t$-test/analysis of variance for quantitative comparisons and $\chi^{2}$ or Fisher's exact test for categorical comparisons.

\section{Data sharing statement}

The CRISPR scores and read counts for gRNA for the CRISPR screen are shown in Supplementary Tables S1 and S7, respectively. The WGS and RNA-seq data that support the findings of this study have been deposited into CNGB Sequence Archive of CNGBdb ${ }^{52}$ with accession number CNP0001595.

\section{Acknowledgements}

We thank B. Zhang for data analysis and contribution to the study and Professor Zubarev's laboratory at KI for sharing anticancer chemicals.

\section{Author details}

${ }^{1}$ Department of Biosciences and Nutrition, Karolinska Institutet, Stockholm, Sweden. ${ }^{2}$ Department of Medical Oncology, Sun Yat-Sen University Cancer Center, State Key Laboratory of Oncology in South China, Collaborative Innovation Center for Cancer Medicine, Guangzhou, China. ${ }^{3}$ Broad Institute of MIT and Harvard, Cambridge, MA, USA. ${ }^{4}$ McGovern Institute for Brain Research, Department of Brain and Cognitive Sciences, and Department of Biological Engineering, Massachusetts Institute of Technology, Cambridge, MA, USA. ${ }^{5}$ Howard Hughes Medical Institute, Cambridge, MA 02139, USA. ${ }^{6}$ BGI-

Shenzhen, Shenzhen 518083, China. ${ }^{7}$ Guangdong Provincial Key Laboratory of Human Disease Genomics, Shenzhen Key Laboratory of Genomics, Shenzhen, China

\section{Author contributions}

M.N. and X.Y. analysed and interpreted the screen data. L.D., M.N., S.C. and X.S. performed the experiments. J.J. helped with the screening experimental design and analysis. X.Y., D.L., W.R. and K.W. analysed the WGS data. F.Z. and Q. P.-H. supervised the study. M.N. and Q.P.-H. wrote the manuscript.

\section{Funding}

This work was supported by the Swedish Cancer Society, the Swedish Research Council, the European Research Council (RNAEDIT-649019), STINT (Joint ChinaSweden mobility programme), Radiumhemmets and the Center for Innovative Medicine (CIMED), Guangdong Enterprise Key Laboratory of Human Disease Genomics (2020B1212070028) and China National GeneBank (CNGB), and the Chinese Natural Science Foundation (81670184). The computational resource was provided by SNIC through Uppsala Multidisciplinary Center for Advanced 
Computational Science (UPPMAX) under project SNIC 2018/8-70. Open access funding provided by Karolinska Institute.

\section{Conflict of interest}

F.Z. is a scientific advisor for Editas Medicine, Beam Therapeutics, Arbor Biotechnologies, Pairwise Plants, and Sherlock Biosciences. F.Z. is also a director for Beam Therapeutics. Other authors declare no competing financial interests.

\section{Publisher's note}

Springer Nature remains neutral with regard to jurisdictional claims in published maps and institutional affiliations.

Supplementary information The online version contains supplementary material available at https://doi.org/10.1038/s41419-021-03695-8.

Received: 24 March 2021 Revised: 7 April 2021 Accepted: 7 April 2021 Published online: 28 April 2021

\section{References}

1. Alizadeh, A. A. et al. Distinct types of diffuse large B-cell lymphoma identified by gene expression profiling. Nature 403, 503-511 (2000).

2. Basso, K. \& Dalla-Favera, R. Germinal centres and B cell lymphomagenesis. Nat Rev. Immunol. 15, 172-184 (2015).

3. Chapuy, B. et al. Molecular subtypes of diffuse large B cell lymphoma are associated with distinct pathogenic mechanisms and outcomes. Nat. Med. 24 679-690 (2018)

4. Schmitz, R. et al. Genetics and pathogenesis of diffuse large B-cell lymphoma. N. Engl. J. Med. 378, 1396-1407 (2018).

5. Lacy, S. E. et al. Targeted sequencing in DLBCL, molecular subtypes, and outcomes: a Haematological Malignancy Research Network report. Blood 135 1759-1771 (2020)

6. Wright, G. W. et al. A probabilistic classification tool for genetic subtypes of diffuse large B cell lymphoma with therapeutic implications. Cancer Cell 37, 551-568 e514 (2020).

7. Ren, W. et al. Genetic landscape of hepatitis B virus-associated diffuse large Bcell lymphoma. Blood 131, 2670-2681 (2018).

8. Shalem, O., Sanjana, N. E. \& Zhang, F. High-throughput functional genomics using CRISPR-Cas9. Nat. Rev. Genet. 16, 299-311 (2015).

9. Wang, $\mathrm{T}$. et al. Identification and characterization of essential genes in the human genome. Science 350, 1096-1101 (2015)

10. Hart, $T$. et al. High-resolution CRISPR screens reveal fitness genes and genotype-specific cancer liabilities. Cell 163, 1515-1526 (2015).

11. Behan, F. M. et al. Prioritization of cancer therapeutic targets using CRISPR-Cas9 screens. Nature 568, 511-516 (2019).

12. Reddy, A. et al. Genetic and functional drivers of diffuse large B cell lymphoma. Cell 171, 481-494 e415 (2017)

13. Phelan, J. D. et al. A multiprotein supercomplex controlling oncogenic signalling in lymphoma. Nature 560, 387-391 (2018).

14. Dersh, D. et al. Genome-wide screens identify lineage- and tumor-specific genes modulating $\mathrm{MHC}-\mathrm{-}$ - and $\mathrm{MHC}-\mathrm{H}$-restricted immunosurveillance of human lymphomas. Immunity 54, 116-131 (2021).

15. Morin, R. D. et al. Somatic mutations altering EZH2 (Tyr641) in follicular and diffuse large B-cell lymphomas of germinal-center origin. Nat. Genet. 42, 181-185 (2010)

16. Morin, R. D. et al. Frequent mutation of histone-modifying genes in nonHodgkin lymphoma. Nature 476, 298-303 (2011).

17. Pasqualucci, L. et al. Inactivating mutations of acetyltransferase genes in B-cell lymphoma. Nature 471, 189-195 (2011).

18. Delvecchio, M., Gaucher, J., Aguilar-Gurrieri, C., Ortega, E. \& Panne, D. Structure of the p300 catalytic core and implications for chromatin targeting and HAT regulation. Nat. Struct. Mol. Biol. 20, 1040-1046 (2013).

19. Kung, A. L. et al. Gene dose-dependent control of hematopoiesis and hematologic tumor suppression by CBP. Genes Dev. 14, 272-277 (2000).

20. Vo, N. \& Goodman, R. H. CREB-binding protein and p300 in transcriptional regulation. J. Biol. Chem. 276, 13505-13508 (2001).

21. Ogiwara, $\mathrm{H}$. et al. Targeting p300 addiction in CBP-deficient cancers causes synthetic lethality by apoptotic cell death due to abrogation of MYC expression. Cancer Discov. 6, 430-445 (2016).
22. Meyer, S. N. et al. Unique and shared epigenetic programs of the CREBBP and EP300 acetyltransferases in germinal center B cells reveal targetable dependencies in lymphoma. Immunity 51, 535-547 e539 (2019).

23. Garbati, M. R., Thompson, R. C., Haery, L. \& Gilmore, T. D. A rearranged EP300 gene in the human B-cell lymphoma cell line RC-K8 encodes a disabled transcriptional co-activator that contributes to cell growth and oncogenicity. Cancer Lett. 302, 76-83 (2011).

24. Kubonishi, I., Niiya, K. \& Miyoshi, I. Establishment of a new human lymphoma line that secretes plasminogen activator. Jpn J. Cancer Res. 76, 12-15 (1985).

25. Guo, $X$. et al. Molecular impact of selective NFKB1 and NFKB2 signaling on DLBCL phenotype. Oncogene 36, 4224-4232 (2017).

26. Mandelbaum, J. et al. BLIMP1 is a tumor suppressor gene frequently disrupted in activated B cell-like diffuse large B cell lymphoma. Cancer Cell 18, 568-579 (2010).

27. Lossos, I. S., Akasaka, T., Martinez-Climent, J. A., Siebert, R. \& Levy, R. The BCL6 gene in B-cell lymphomas with 3q27 translocations is expressed mainly from the rearranged allele irrespective of the partner gene. Leukemia 17, 1390-1397 (2003).

28. Schneider, B. et al. T(3;7)(q27;q32) fuses BCL6 to a non-coding region at FRA7H near miR-29. Leukemia 22, 1262-1266 (2008).

29. Haery, L., Lugo-Pico, J. G., Henny, R. A., Andrews, A. J. \& Gilmore, T. D. Histone acetyltransferase-deficient p300 mutants in diffuse large B cell lymphoma have altered transcriptional regulatory activities and are required for optimal cell growth. Mol. Cancer 13, 29 (2014).

30. Tzelepis, K. et al. A CRISPR dropout screen identifies genetic vulnerabilities and therapeutic targets in acute myeloid leukemia. Cell Rep. 17, 1193-1205 (2016).

31. Tsherniak, A. et al. Defining a cancer dependency map. Cell 170, 564-576 e516 (2017).

32. Meyers, R. M. et al. Computational correction of copy number effect improves specificity of CRISPR-Cas9 essentiality screens in cancer cells. Nat. Genet. 49 1779-1784 (2017).

33. Trabucco, S. E. et al. Inhibition of bromodomain proteins for the treatment of human diffuse large B-cell lymphoma. Clin. Cancer Res. 21, 113-122 (2015).

34. Lasko, L. M. et al. Discovery of a selective catalytic p300/CBP inhibitor that targets lineage-specific tumours. Nature 550, 128-132 (2017).

35. Zhang, J. et al. The CREBBP acetyltransferase is a haploinsufficient tumor suppressor in B-cell lymphoma. Cancer Discov. 7, 322-337 (2017).

36. Conery, A. R. et al. Bromodomain inhibition of the transcriptional coactivators CBP/EP300 as a therapeutic strategy to target the IRF4 network in multiple myeloma. Elife 5, e10483 (2016).

37. Neuveut, C., Wei, Y. \& Buendia, M. A. Mechanisms of HBV-related hepatocarcinogenesis. J. Hepatol. 52, 594-604 (2010)

38. Kandoth, $\mathrm{C}$. et al. Mutational landscape and significance across 12 major cancer types. Nature 502, 333-339 (2013).

39. Wade, M., Li, Y. C. \& Wahl, G. M. MDM2, MDMX and p53 in oncogenesis and cancer therapy. Nat. Rev. Cancer 13, 83-96 (2013).

40. Stolte, B. et al. Genome-scale CRISPR-Cas9 screen identifies druggable dependencies in TP53 wild-type Ewing sarcoma. J. Exp. Med. 215, 2137-2155 (2018).

41. Haapaniemi, E., Botla, S., Persson, J., Schmierer, B. \& Taipale, J. CRISPR-Cas9 genome editing induces a p53-mediated DNA damage response. Nat. Med. 24, 927-930 (2018).

42. Li, W. et al. MAGeCK enables robust identification of essential genes from genome-scale CRISPR/Cas9 knockout screens. Genome Biol. 15, 554 (2014).

43. Mertins, P. et al. Proteogenomics connects somatic mutations to signalling in breast cancer. Nature 534, 55-62 (2016).

44. Pastore, $A$. et al. Integration of gene mutations in risk prognostication for patients receiving first-line immunochemotherapy for follicular lymphoma: a retrospective analysis of a prospective clinical trial and validation in a population-based registry. Lancet Oncol. 16, 1111-1122 (2015).

45. Chen, $X$. et al. Manta: rapid detection of structural variants and indels for germline and cancer sequencing applications. Bioinformatics 32, 1220-1222 (2016).

46. Ye, X., et al. Genome-wide mutational signatures revealed distinct developmental paths for human B cell lymphomas. J. Exp. Med. 218, e20200573 (2021).

47. Boeva, V. et al. Control-FREEC: a tool for assessing copy number and allelic content using next-generation sequencing data. Bioinformatics 28, 423-425 (2012).

48. Joung, J. et al. Genome-scale CRISPR-Cas9 knockout and transcriptional activation screening. Nat. Protoc. 12, 828-863 (2017). 
49. Langmead, B. \& Salzberg, S. L. Fast gapped-read alignment with Bowtie 2. Nat. Methods 9, 357-359 (2012)

50. Subramanian, A. et al. Gene set enrichment analysis: a knowledge-based approach for interpreting genome-wide expression profiles. Proc. Natl Acad. Sci. USA 102, 15545-15550 (2005).
51. Liberzon, A. et al. The Molecular Signatures Database (MSigDB) hallmark gene set collection. Cell Syst. 1, 417-425 (2015).

52. Guo, X., et al. CNSA: a data repository for archiving omics data. Database 2020 baaa055 (2020) 